\title{
Diagnóstico por imagen de la disección arterial coronaria espontánea
}

\author{
Javier Ibero Valencia* \\ Ana De la Fuente* \\ José Calabuig Nogués*
}

\author{
Juan José Gavira Gómez* \\ Gorka Bastarrika Alemañ ${ }^{* *}$
}

\section{Correspondencia}

Javier Ibero Valencia

jibero.1@unav.es

\begin{abstract}
* Departamento de Cardiología y Cirugía Cardiovascular. Clínica Universidad de Navarra. España
** Servicio de Radiología. Clínica Universidad de Navarra. España

\section{Recibido: 06/03/2020 \\ Aceptado: 28/05/2020 \\ En línea: 31/07/2020}

Citar como: Ibero-Valencia J, De la Fuente A, Calabuig-Nogués J, Gavira-Gómez JJ, Bastarrika-Alemañ G. Diagnóstico por imagen de la disección arterial coronaria espontánea. Rev Ecocar Pract (RETIC). 2020 (Jul); 3 (2): 14-17. doi: 10.37615/retic.v3n2a5.

Cite this as: Ibero-Valencia J, De la Fuente A, Calabuig-Nogués J, Gavira-Gómez JJ, Bastarrika-Alemañ G. Imaging diagnosis of spontaneous coronary artery dissection. Rev Ecocar Pract (RETIC). 2020 (Jul); 3 (2): 14-17. doi: 10.37615/retic.v3n2a5.

\section{Palabras clave}

$\triangleright$ Síndrome coronario agudo

$\triangleright$ Disección coronaria espontánea

$\triangleright$ Diagnóstico

\section{RESUMEN}

La disección coronaria espontánea (DCE) es una causa infrecuente de síndrome coronario agudo (SCA) que precisa una aproximación diagnóstica y terapéutica específica. La coronariografía invasiva es la técnica de elección para el diagnóstico de la DCE. Sin embargo, la incapacidad para caracterizar la pared arterial hace necesario, en algunos casos, utilizar otras técnicas diagnósticas, como la imagen intracoronaria o la coronariografía porTC. Se presenta el caso de una mujer joven, puérpera, con diagnóstico de preeclampsia, que desarrolla un SCA secundario a una DCE donde se demuestra la utilidad de las distintas técnicas diagnósticas.

ABSTRACT
Spontaneous coronary artery dissection (SCAD) is an infrequent cause of acute coronary syndrome (ACS), which
requires a specific diagnostic workup and therapeutic management. Coronary angiography is the preferred te-
chnique for the diagnosis of SCAD. However, its inability to characterize the artery wall emphasizes the need of
other imaging techniques, such as intracoronary imaging or coronary CT angiography. We present a case report
of a young postpartum woman with preeclampsia who developed an ACS due to a spontaneous coronary artery
dissection. In this case we demonstrate the clinical value of different imaging techniques.

\section{Presentación del caso}

Se presenta el caso de una mujer puérpera de 35 años hospitalizada en el centro de los autores por preeclampsia en el posparto inmediato. Entre sus antecedentes médicos destacaba el diagnóstico de hipertensión arterial y el desarrollo de preeclampsia en dos embarazos previos.

A los 7 días de dar a luz a su tercer hijo, presentó un episodio de opresión precordial en reposo coincidiendo con pico hipertensivo de 200/120 mmHg. Por ello se le realizó un electrocardiograma urgente que, comparado con su electrocardiograma basal, mostró rectificación del segmento ST en V4-V6, así como descenso del ST e inversión de ondas T en dIII y aVF (Figura 1). Se completó con estudio analítico que puso de manifiesto elevación de enzimas cardíacas con curva isquémica.

Ante estos hallazgos se procedió a la realización de un cateterismo urgente que mostró imagen de disección extraluminal del origen de la arteria coronaria posterolateral, así como reducción brusca y homogénea del calibre de la des- cendente anterior desde su tercio medio y de la primera diagonal (Figura 2). Se completó el estudio con IVUS que evidenció hematoma intramural en ambas arterias (Vídeo 1). Ante estos hallazgos la paciente fue diagnosticada de disección coronaria espontánea.

Dada la estabilidad hemodinámica de la paciente se decidió mantener una actitud expectante. Al día siguiente se le realizó una coronariografía por TC, que mostró imágenes compatibles con hematoma intramural correspondiente con los hallazgos de la coronariografía invasiva (Figura 3 a Figura 5), así como un área de hipoperfusión e hipocinesia del segmento anterior apical correspondiente con el territorio de la primera diagonal (Figura 6 y Figura 7).

La paciente presentó buena evolución posterior y fue dada de alta asintomática desde el punto de vista cardiovascular.

A los 3 meses acudió a revisión encontrándose asintomática desde el punto de vista cardiovascular. Se la realizó una nueva coronariografía por TC, que evidenció la resolución del hematoma intramural (Figura 8 y Figura 9). 


\section{Estudio por imagen}

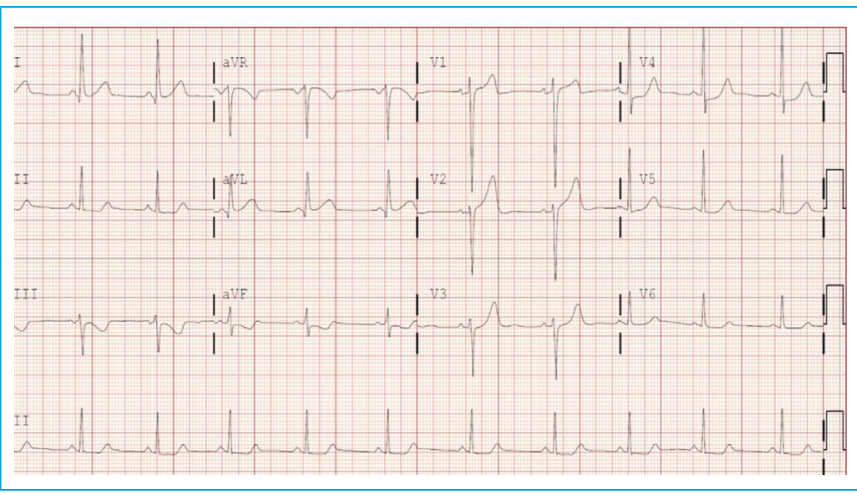

Figura 1. Electrocardiograma. Rectificación del segmento ST en V4-V6, así como descenso del ST e inversión de ondas T en dIIll y aVF
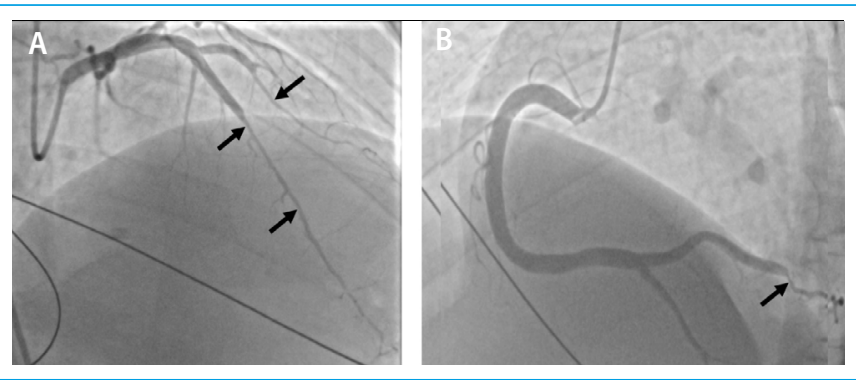

Figura 2. Coronariografía invasiva. Se evidencia reducción brusca y homogénea del calibre de la descendente anterior desde su tercio medio (flecha negra) y de la primera diagonal (A), así como posible disección extraluminal del origen de la arteria coronaria posterolateral (B)

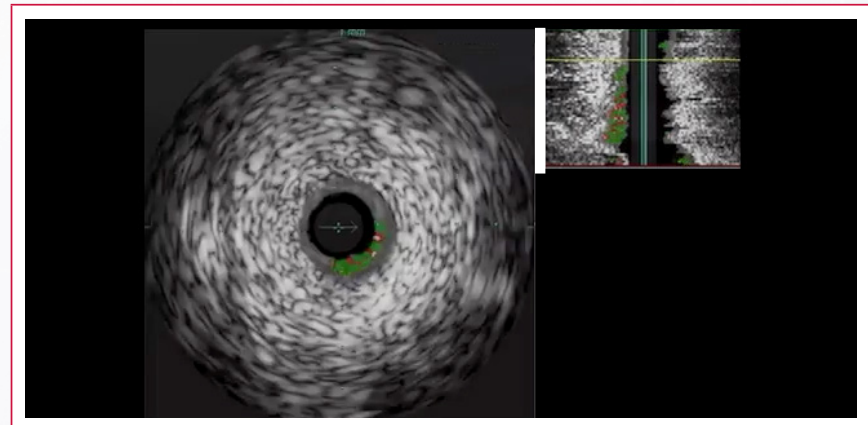

Vídeo 1. IVUS. Se observa hematoma intramural desde el tercio medio de la arteria descendente anterior
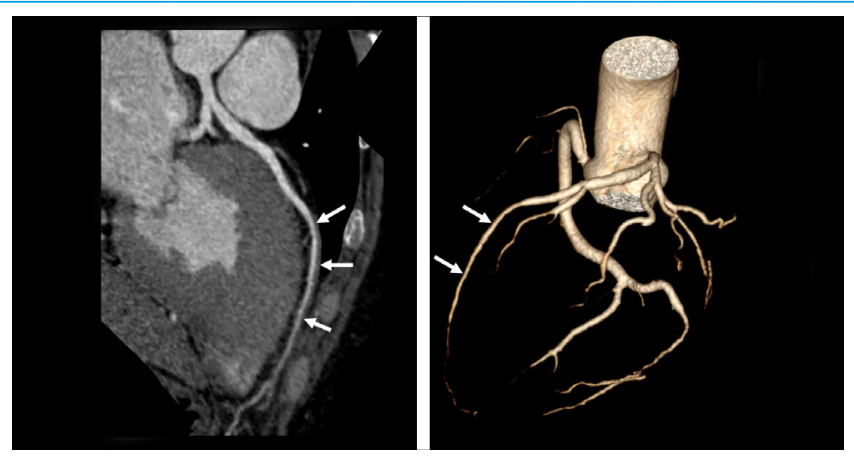

Figura 3. Coronariografía por TC, estudio basal. Se evidencia hematoma intramural en la descendente anterior media, concordante con las imágenes del IVU

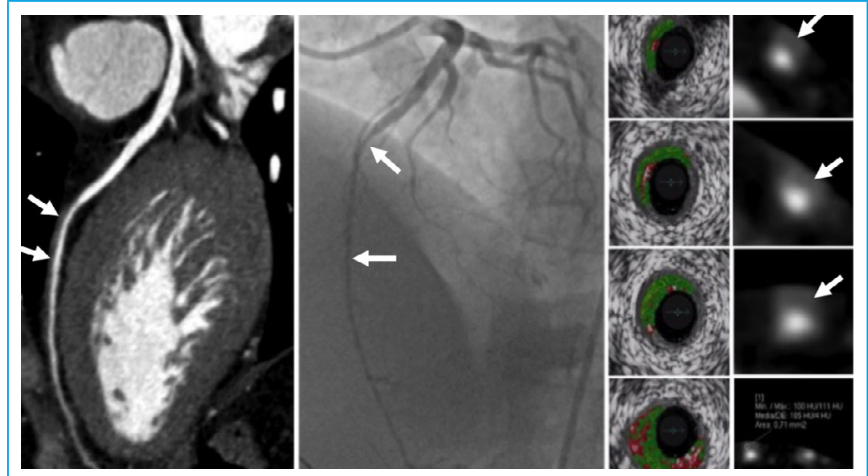

Figura 4. Comparativa de los hallazgos presentes en la coronariografía por TC, la coronariografía invasiva y los presentes en el IVUS
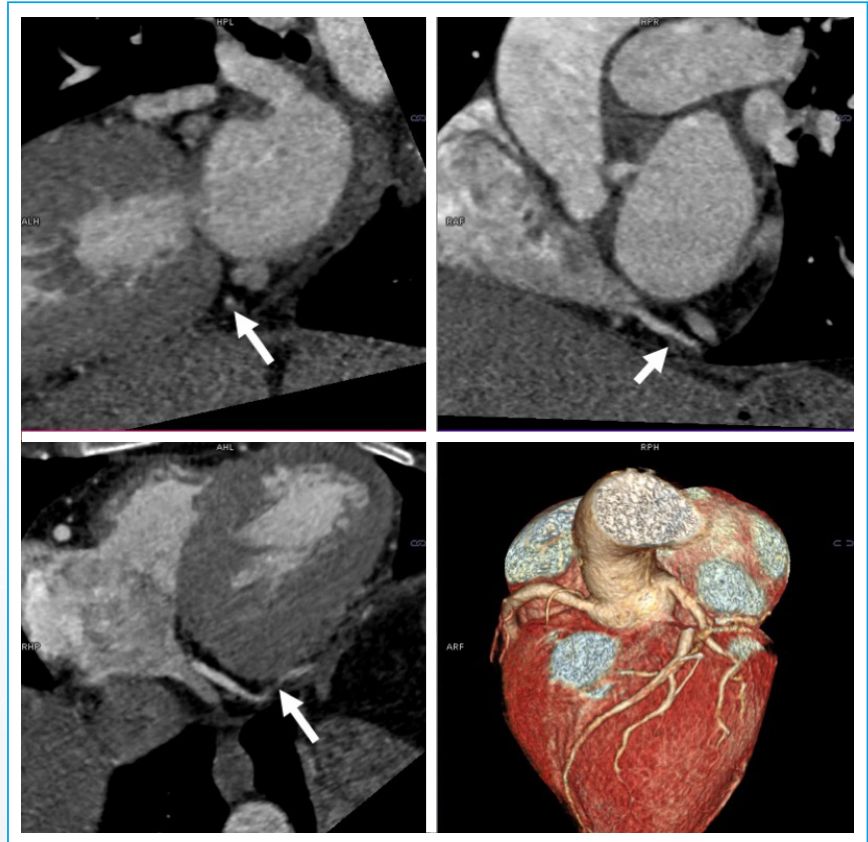

Figura 5. Coronariografía por TC, estudio basal. Se evidencia hematoma intramural en la posterolateral, concordante con las imágenes del IVUS
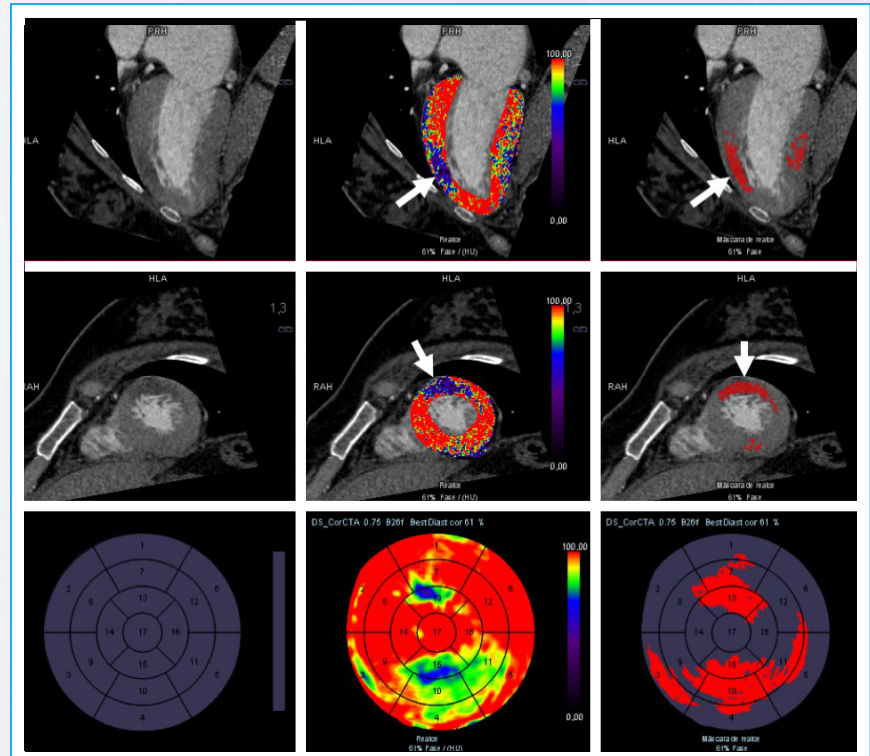

Figura 6. Estudio de perfusión miocárdica por TC. Se observa hipoperfusión de los segmentos anterior e inferior apical del ventrículo izquierdo 


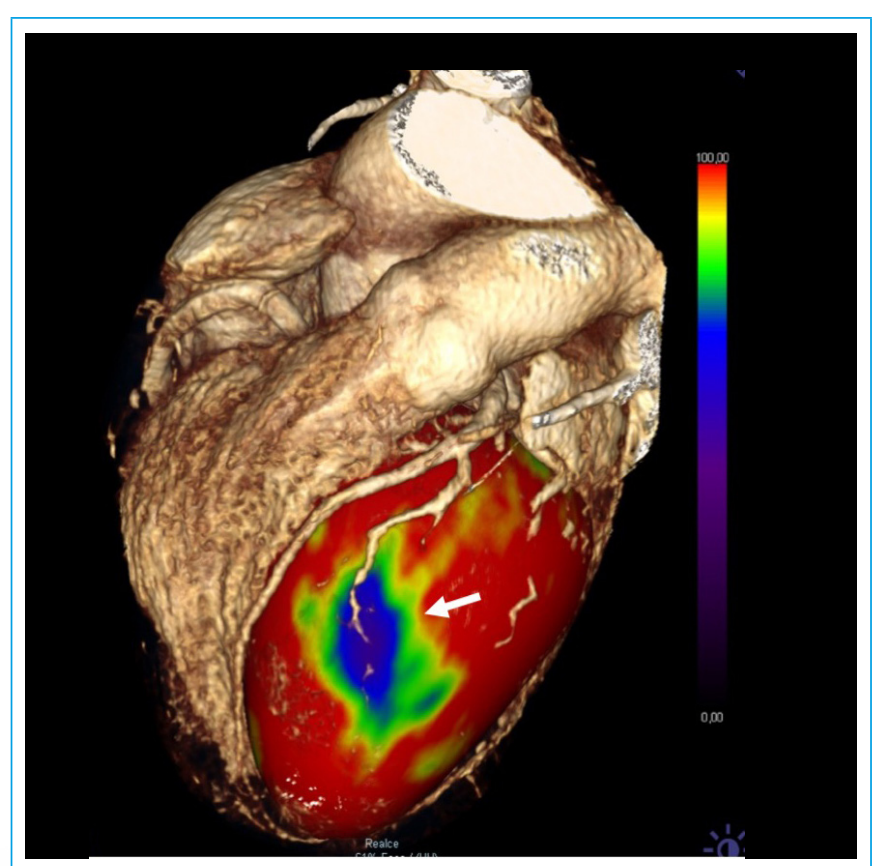

Figura 7. Estudio de perfusión miocárdica por TC. Reconstrucción tridimensional con visualización del déficit de perfusión en cara anterior

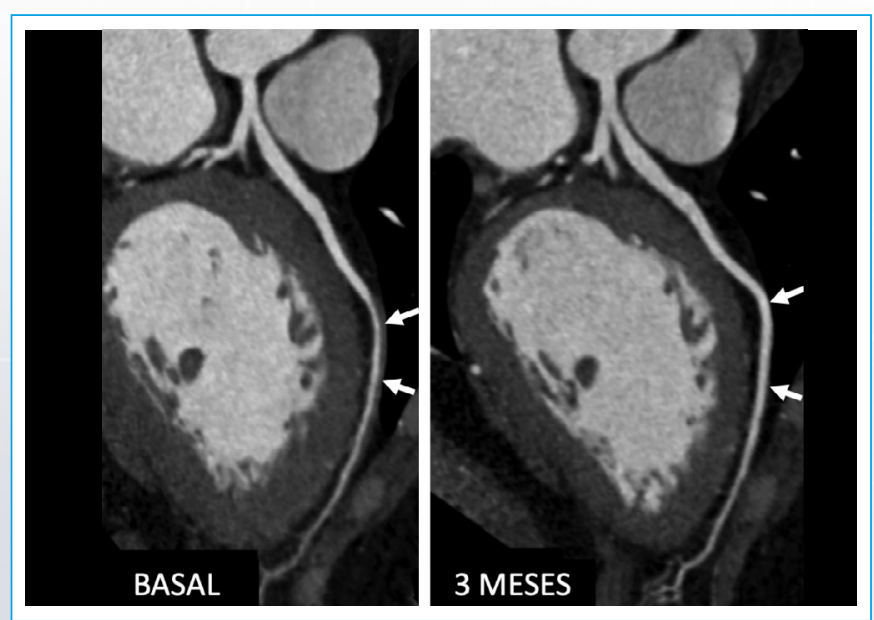

Figura 8. Comparación de coronariografía por TC del estudio basal con el control a los 3 meses (arteria coronaria descendente anterior)

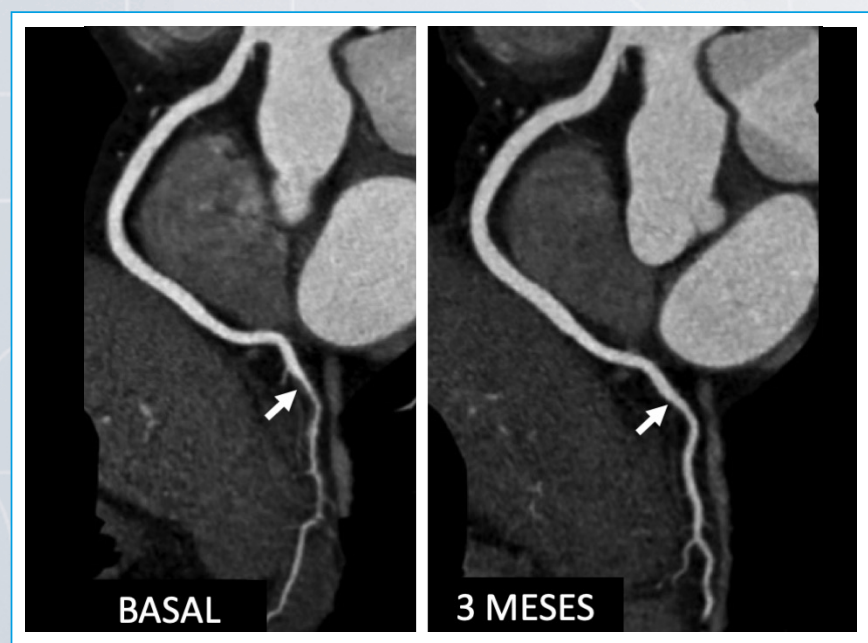

Figura 9. Comparación de coronariografía por TC del estudio basal con el control a los 3 meses (rama posterolateral)

\section{Discusión}

La disección coronaria espontánea (DCE) es una separación de las capas que conforman la pared de la arteria coronaria secundaria a la formación de un hematoma intramura ${ }^{(1,2)}$. Esta entidad se puede asociar o no a una rotura de la íntima y no debe ser secundaria a iatrogenia o traumatismo. La compresión extrínseca de la luz verdadera puede ser causa de síndrome coronario agudo (SCA).

Siendo una causa infrecuente de SCA, clásicamente la DCE se ha asociado a mujeres jóvenes embarazadas. Sin embargo, aunque constituye la principal causa de SCA durante el embarazo, esta asociación solamente está presente en un 10\% del total. La arteria coronaria que más frecuentemente se afecta es la descendente anterior y en hasta un $23 \%$ puede presentar afectación multivaso(3).

Para su diagnóstico se precisa una alta sospecha diagnóstica con objeto de orientar como SCA a un paciente con dolor torácico con escasa probabilidad pre-test de cardiopatía isquémica ateroesclerótica ${ }^{(1,4)}$. La presencia de un dolor torácico típico en el contexto de una mujer joven ( $<50$ años) con pocos o ningún factor de riesgo cardiovascular, antecedente de parto reciente o arteriopatías genéticas como el síndrome de Marfan debe elevar la sospecha diagnóstica de esta entidad.

La técnica diagnóstica de elección es la coronariografía invasiva, si bien es cierto que tiene limitaciones, puesto que no es capaz de caracterizar la pared de la arteria y, por tanto, de identificar el hematoma intramural ${ }^{(1)}$. Por ello, las técnicas de imagen intracoronaria, como el IVUS o la OCT, constituyen herramientas fundamentales para la identificación de dicho hematoma. En este caso, la paciente presentó una disección tipo $2 \mathrm{~b}^{(5)}$, y se precisó la realización de un IVUS intracoronario con objeto de confirmar el diagnóstico de la enfermedad.

La coronariografía por TC (CTC) es una técnica no invasiva capaz de valorar la anatomía coronaria. Sin embargo, su resolución espacial es relativamente baja, por lo que esta técnica presenta dificultades para identificar la enfermedad (ya sea en forma de oclusión, disección o hematoma intramural) en los vasos de menor calibre. Por este motivo la ausencia de disección en la CTC no excluye el diagnóstico de disección coronaria ${ }^{(6)}$. En este caso se realizó una CTC 24 horas después de la coronariografía invasiva, haciéndose evidente la presencia de hematoma intramural, tanto en el tercio medio de la descendente anterior como en la primera diagonal y en la arteria posterolateral. Además, la CTC también permite establecer la perfusión del miocardio, como en este caso, en el que se observó un déficit de perfusión en el territorio de la primera diagonal.

A pesar de sus limitaciones, la CTC puede ser útil en el seguimiento de aquellos pacientes con CTC patológica. Hasta en un 90\% de los casos el hematoma intramural se resuelve de forma espontánea ${ }^{(7)}$. Esto justifica que, excepto en aquellos casos en los que se demuestre isquemia persistente o inestabilidad hemodinámica, no se utilicen técnicas de revascularización. En este contexto, la CTC es especialmente útil para demostrar de forma no invasiva la resolución del cuadro. En este caso particular la arteria estaba permeable en el momento de la coronariografía invasiva, sin signos de isquemia clínica o electrocardiográfica tras el inicio de vasodilatadores, motivo por el que no se revascularizó. En la CTC a los 3 meses se demostró la resolución del hematoma intramural.

\section{Conclusión}

La DCE es una causa infrecuente de SCA, que precisa un manejo diagnóstico y terapéutico específico. La coronariografía invasiva y las técnicas de imagen intracoronaria constituyen el pilar diagnóstico de esta entidad, mientras que la coronariografía por TC es una técnica útil en el seguimiento a largo plazo de estos pacientes. 


\section{Ideas para recordar}

- La disección arterial coronaria es una causa infrecuente de síndrome coronario agudo, por lo que se precisa una alta sospecha diagnóstica en pacientes con un perfil clínico determinado para diagnosticarla.

- La coronariografía invasiva y las técnicas de imagen intracoronaria son las técnicas de elección en el diagnóstico de esta entidad.

- La coronariografía por TC, a pesar de sus limitaciones en el diagnóstico, puede ser de utilidad en el seguimiento no invasivo de la enfermedad.

\section{Bibliografía}

1. Hayes SN, Kim ESH, Saw J, et al. Spontaneous coronary artery dissection: current state of the science: a scientific statement from the American Heart Association. Circulation 2018; 137 (19): e523-e557.
2. Adlam D, Alfonso F, Maas AH, et al. European Society of Cardiology, acute cardiovascular care association, SCAD study group: a position paper on spontaneous coronary artery dissection. Eur Heart J 2018; 39 (36): 3353-3368.

3. Tweet MS, Hayes SN, Gulati R, et al. Clinical features, management, and prognosis of spontaneous coronary artery dissection. Circulation 2012; 126 (5): 579-588.

4. Adlam D, García-Guimaraes M, Maas AHEM. Spontaneous coronary artery dissection: no longer a rare disease. Eur Heart J 2019; 40 (15): 1198-1201.

5. Saw J. Coronary angiogram classification of spontaneous coronary artery dissection. Catheter Cardiovasc Interv 2014; 84 (7): 1115-1122.

6. Tweet MS, Akhtar NJ, Hayes SN, et al. Spontaneous coronary artery dissection: Acute findings on coronary computed tomography angiography. Eur Heart J Acute Cardiovasc Care 2019; 8 (5): 467-475.

7. Saw J, Starovoytov A, Humphries K, et al. Canadian spontaneous coronary artery dissection cohort study: in-hospital and 30-day outcomes. Eur Heart J 2020; 40 (15): 1188-1197. 